\section{Athero-inflammation link crystallizes}

\author{
By Lev Osherovich, Senior Writer
}

Cholesterol-rich arterial plaques have long been known to harbor proinflammatory immune cells such as macrophages, but the order of events leading to full-blown atherosclerosis has been unclear. Now, a study by German and American researchers provides a mechanism for the long-suspected link between inflammation and atherosclerosis. The findings implicate a crystalline form of cholesterol as a trigger for production of the proinflammatory cytokine IL-1 $\beta{ }^{1}$

The results could open atherosclerosis as a new indication for IL- $1 \beta$ antagonists that are being developed by Xoma Ltd., Swedish Orphan Biovitrum and Novartis AG.

"We have found one of the most important stimulators in atherosclerosis. Everybody knows that cholesterol is bad, but we have shown that it's specifically the crystalline form of cholesterol that's responsible" for the inflammatory component of atherosclerosis, said the study's senior author, Eicke Latz, assistant professor of infectious diseases and immunology at the University of Massachusetts Medical School and director of the Institute of Innate Immunity at the University of Bonn.

Latz's study suggests that cholesterol crystals are "a danger signal that the body seeks to eliminate, akin to uric acid crystals in gout," said Stephen James, VP and head of research at Biovitrum. The report suggests that atherosclerosis "can be added to the burgeoning list of autoinflammatory diseases."

\section{Inflammation trigger}

Latz's team discovered the role of cholesterol crystals by feeding atherosclerosis-prone mice a cholesterol-rich diet and monitoring the growth of atherosclerotic plaques in the aorta. The team noticed that crystal-like structures appeared early in the course of disease, before the accumulation of innate immune cells in full-blown plaques.

Similar-looking crystals of metabolites such as uric acid and calcium pyrophosphate are recognized by the innate immune system as foreign particles, leading to inflammatory conditions such as gout. Innate immune cells respond to foreign particles by means of the inflammasome, a proteolytic complex that converts IL- $1 \beta$ into an active form. ${ }^{2}$ Thus, Latz suspected that the inflammasome also could be activated by cholesterol crystals.

Indeed, cultured human cells treated with cholesterol crystals had active inflammasomes and secreted IL-1 $\beta$, whereas mock-treated controls did not. Blocking inflammasome activation also inhibited IL-1 $\beta$ production in response to cholesterol crystals.

The team then went back into mice and found that peritoneal injection of cholesterol crystals led to inflammation and aortic atherosclerosis. However, knocking out various components of the inflammasome blocked the harmful effect of injected cholesterol crystals.

That result suggested the inflammasome is needed to initiate fullblown atherosclerosis in response to the crystals.

The results were reported in Nature.

\section{Crystal clear}

The paper offers several new directions for development of atherosclerosis therapeutics. The findings could provide a clue about the mechanism of the suspected anti-inflammatory effect of statin treatment, suggesting a more specific focus on crystal formation.

At present, clinicians try to prevent the formation of atherosclerotic plaques by inhibiting cholesterol biosynthesis with statins. However, it's unclear whether lowering cholesterol would be sufficient to downregulate immune cells already activated by cholesterol crystals.

"That cholesterol crystals can form quickly in early atherosclerotic lesions in experimental animal models is remarkable," said Ta Yuan Chang, chair of biochemistry at Dartmouth College. "It would now be important to understand how these cholesterol crystals are formed and what roles they play in different stages of atherosclerosis."

Latz suggested that statins may exert their effect on atherosclerosis by reducing cholesterol below the threshold needed to form crystals. "It needs to be determined whether just controlling cholesterol levels can reduce crystal formation," he said.

Meanwhile, the Latz team is exploring how the crystals activate innate immunity, hoping to uncover an angle on how to block the inflammatory cascade that may lead to heart disease. The group is running a screen for small molecule inflammasome inhibitors and hopes to test them in atherosclerosis models.

The Nature study further implies that mAbs that reduce IL- $1 \beta$ activity could be effective in atherosclerosis.

Alan Solinger, VP of clinical immunology at Xoma, said IL- $1 \beta$ has turned up as a critical mediator of inflammation in sports injuries, which can be exacerbated by cholesterol crystal accumulation in joints.

"There's clinical evidence of the crystal nature of inflammation initiation" in other tissues, said Solinger, who said the breakthrough in Latz's study is the link between the crystals and the inflammasome.

Solinger said preclinical studies showed that XOMA-052 reduced symptoms of type 2 diabetes and metabolic syndromes. The mAb also reduced atherosclerosis in experiments with the same mouse model used by Latz's team.

XOMA-052, an IL-1 $\beta$-blocking $\mathrm{mAb}$, is in Phase II trials for type 2 diabetes. 


\section{ANALYSIS/PROSPECTS}

Ilaris canakinumab, an anti-IL-1 $\beta$ mAb from Novartis, also could potentially be tested as an atherosclerosis therapy. Ilaris is in Phase III testing for cryopyrin-associated periodic syndrome (CAPS), a rare hereditary autoimmune disease caused by excessive inflammasome activity.

Another option might be to block IL- $1 \beta$ further downstream at the IL-1 receptor 1 (IL1R1; CD121A), which is the target of Swedish Orphan Biovitrum's Kineret anakinra. Kineret, which the company exclusively licensed from Amgen Inc., is marketed to treat rheumatoid arthritis (RA).

One concern about using IL- $1 \beta$-blocking mAbs to treat atherosclerosis, James noted, is that systemically administered biologics may have a harder time penetrating the core of mature plaques than small molecules would.

Solinger, James and Chang agreed that targeting IL-1 $\beta$ would most likely be an adjunct to conventional cholesterol-lowering statin therapy. "Statins will always be first-line therapy," said James. "Blocking IL-1 $\beta$ could be a complementary treatment in those people [in whom] the effects of statins are insufficient."

"Combining anti-IL-1 $\beta$ therapy with a statin could be synergistic," said Solinger. "You could potentially lower the dose of statin."

Latz said the University of Massachusetts has filed patents on the discoveries, which are available for licensing.

Osherovich, L. SciBX 3(18); doi:10.1038/scibx.2010.543

Published online May 6, 2010

\section{REFERENCES}

1. Duewell, P. et al. Nature; published online April 29, 2010; doi:10.1038/nature08938

Contact: Eicke Latz, University of Massachusetts Medical School, Worcester, Massachusetts

e-mail: eicke.latz@uni-bonn.de or eicke.latz@umassmed.edu

2. Schroder, K. et al. Science 327, 296-300 (2010)

\section{COMPANIES AND INSTITUTIONS MENTIONED}

Amgen Inc. (NASDAQ:AMGN), Thousand Oaks, Calif.

Dartmouth College, Hanover, N.H.

Novartis AG (NYSE:NVS; SIX:NOVN), Basel, Switzerland

Swedish Orphan Biovitrum (SSE:BVT), Stockholm, Sweden

University of Massachusetts Medical School, Worcester, Mass.

University of Bonn, Bonn, Germany

Xoma Ltd. (NASDAQ:XOMA), Berkeley, Calif. 